\title{
Ets-1 Targeted by MicroRNA-221 Regulates Angiotensin II-Induced Renal Fibroblast Activation and Fibrosis
}

\author{
Jia Dia ${ }^{a, b}$ Lei Jiang ${ }^{a}$ Yang Zhou Hongdi Cao $^{a}$ Li Fang ${ }^{a}$ Ping Wen ${ }^{a}$ Xiurong Lib \\ Chunsun Daia Junwei Yanga
}

${ }^{a}$ Center for Kidney Disease, $2^{\text {nd }}$ Affiliated Hospital, Nanjing Medical University, Nanjing, ${ }^{b}$ Departments of Nephrology, $3^{\text {rd }}$ Affiliated Hospital, Soochow University, Changzhou, China

\author{
Key Words \\ Angiotensin II • Fibroblast • Renal fibrosis • microRNA
}

\begin{abstract}
Background: Fibroblast activation is one of the most important mechanisms for Angiotensin II (Ang II) in promoting renal fibrosis. Transcription factor Ets-1 is recognized to play a key role in kidney diseases. However, the role and mechanisms of Ets-1 in Ang-II induced fibroblast activation and kidney fibrosis are not fully understood. Methods: Mice were treated with Ang II via osmotic mini-pumps or Ang II expression plasmid (pAng II). Cultured normal rat kidney interstitial fibroblast (NRK-49F) cells were incubated with Ang II. Role of Ets-1 in renal fibrosis and fibroblast activation were assessed by Western blot, Immunohistochemical staining,MTT, Boyden chamber and Immunofluorescence staining. Effects of miR-221 on Ets-1 and fibroblast activation were investigated by MTT, Boyden chamber, Western blot and Q-PCR. Results: We found that Ets-1 was up-regulated in fibrotic kidneys. Similarly, Ang II could activate NRK-49F cells as demonstrated by up-regulated $\alpha$-SMA and fibronectin(FN) expression and enhanced cell proliferation and migration. Ang II also induced Ets-1 expression in NRK-49F cells in a dose and time dependent manner. Knock-down of Ets-1 by RNA interference attenuated Ang IIinduced activation of NRK-49F cells. Ets- 1 was previously reported as a target of microRNA-221 (miR-221). In Ang II-induced fibrotic kidney, miR-221 was down-regulated. Similar results were observed in Ang II treated NRK-49F cells. Ectopic expression of miR-221 mimic attenuated the up-regulation of Ets-1 by Ang II in NRK-49F cells, which further prevented the activation of NRK-49F cells. However, the inhibitor of miR-221 aggravated Ang II induced Ets-1 expression and NRK-49F cells activation. Conclusions: Our study suggests that miR-221/Ets-1 axis takes an important role in mediating AngII induced interstitial fibroblast activation and renal fibrosis.
\end{abstract}

Copyright (C) 2014 S. Karger AG, Basel 


\section{Cellular Physiology and Biochemistry}

Cell Physiol Biochem 2014;34:1063-1074

\begin{tabular}{l|l}
\hline DOI: $10.1159 / 000366321$ & (c) 2014 S. Karger AG, Basel
\end{tabular}

Published online: september 08, 2014

www.karger.com/cpb

Di et al.: miR-221 Antagonizes Ang II-Induced Renal Fibrosis by Targeting Ets-1

\section{Introduction}

The prevalence of CKD is increasing worldwide [1,2]. Renal interstitial fibrosis is an inevitable result of virtually all progressive kidney diseases. Solid evidence has pointed out that the key mediator of fibrotic tissue remodeling is the activation of ECM-producing myofibroblasts [3], which originated from local interstitial fibroblasts, pericytes, bone marrow-derived circulating fibrocytes, local mesenchymal stem cells, endothelium, and injured epithelium [4]. Among them, activation of local interstitial fibroblasts have been recognized as the majority contributor [5]. Under physiological conditions, fibroblasts stay in the kidney interstitium and maintain the homeostasis of interstitial matrix and adjacent tissue. Once activated, they acquire the myofibroblast phenotype characterized by $\alpha$-SMA expression and other markers [5-7]. These activated fibroblasts may proliferate and invade the periglomerular and peritubular spaces, contributing to matrix deposition in the tubulointerstitial area. Mechanical stress, cytokines and various other factors have been identified to induce fibroblasts activation $[8,9]$. However, the intracellular molecular mechanisms regulating fibroblast activation remain largely unknown.

Ang II emerges as a major profibrogenic cytokine of tubulointerstitial fibrosis. Emerging evidences have shown that AngII could upregulate many profibrotic factors such as TGF- $\beta 1$, CTGF [10], induce epithelial mesenchymal transition, activate inflammatory cells and produce extracellular matrix [11]. One of the most important mechanisms is stimulating fibroblast activation. Ets-1 is an important member of the Ets transcription factor family that participates in the regulation of a wide variety of biological processes [12-14]. Previous investigations demonstrated that Ets-1 participated in Ang II-induced ROS generation and inflammation $[15,16]$. However, the role and mechanisms of Ets-1 in Ang-II induced fibroblast activation and kidney fibrosis are still not understood.

MicroRNAs (miRNAs) are a family of endogenous noncoding RNA with 20-22 nucleotides in length. They have been proved to induce degradation or repress translation of target mRNAs. It is reported that up to $30 \%$ of mammalian protein-encoding genes are the targets of miRNAs [17]. Recent investigations demonstrated that Ets-1 was a target of miR-221 in Ang II-induced endothelial inflammation and migration [18]. Large amounts of data demonstrated that miR-221 regulated various kinds of cancer [19-22]. However, the role of miR-221 in renal fibrosis remains unclear. It has been reported that miR-221 was downregulated in fibrotic kidney after unilateral ureteral occlusion (UUO) [23]. In this study, we detected miR-221 expression in Ang II-induced fibrotic kidney and Ang II treated NRK49F cells. Consist with miR-221 was downregulated after UUO, we found that miR-221 was decreased while Ets- 1 was upregulated after AngII infusion. In cultured NRK-49F cells, AngII could also downregulate miR-221 and increase Ets-1 expression. We further demonstrated that miR-221 regulated Ets-1 expression and involved in AngII induced fibroblast activation and renal fibrosis.

\section{Materials and Methods}

\section{Ethics statement}

All of the following details of the study were approval by the responsible ethics committee of Nanjing Medical University (Permit Number: KY2012020). The written informed consent was supplied by the patients before the study.

The animal ethics approval was obtained from the Animal Care and Use Committee of Nanjing Medical University. All experiments were conducted in accordance with the Guide for the Care and Use of Laboratory Animals adopted by the Institutional Animal Care and Use Committee (IACUC) to ensure the proper care and welfare of animals involved in research. All the mice were housed in single cages and the housing room was maintained at a $12 \mathrm{~h}$ light/dark cycle (lights on at $8.00 \mathrm{~h}$ ). Food and water were provided ad libitum. Mice were sacrificed under anesthesia. All the animal experiments were made to minimize suffering and reduce the number of animals used. 


\section{Cellular Physiology and Biochemistry}

Cell Physiol Biochem 2014;34:1063-1074

\begin{tabular}{l|l}
\hline DOI: $10.1159 / 000366321$ & (c) 2014 S. Karger AG, Basel
\end{tabular}

www.karger.com/cpb

Di et al.: miR-221 Antagonizes Ang II-Induced Renal Fibrosis by Targeting Ets-1

Animal models

Male CD1 or female Balb/c mice that weighed 20-22 g were acquired from the Specific Pathogen-Free Laboratory Animal Center of Nanjing Medical University. They were housed in the animal facilities of the Kidney Disease Center of the 2nd Affiliated Hospital of Nanjing Medical University. Animals were treated humanely in accordance with NMAC guidelines and approved protocols of the Nanjing Medical University Institutional Animal Use and Care Committee. Ang II (sigma) was infused at the rate of $1 \mu \mathrm{g} / \mathrm{kg} / \mathrm{min}$ by a miniosmotic pump (Alzet) for 4 weeks in male CD1 mice. The Ang II expression plasmid (pAng II) was injected intravenously at $1 \mathrm{mg} / \mathrm{kg}$ of body weight using a hydrodynamics-based in vivo gene transfer approach in female Balb/c mice. To euthanize the mice, pentobarbital sodium (150-200 mg/kg, intraperitoneally) was used at 4- weeks after the treatment; serum and urine were collected and the kidneys were harvested.

\section{Cell culture and treatment}

The rat renal interstitial fibroblast (NRK-49F) cells were obtained from American Type Culture Collection(Manassas, VA, USA). Cells were cultured in DMEM/F12 medium supplemented with 10\% fetal bovine serum(Invitrogen, San Diego, CA). For Ang II treatment, NRK-49F cells were seeded at 70\% confluence in complete medium containing $10 \%$ fetal bovine serum. Twenty-four hours later, the cells were changed to serum-free medium and incubated for $16 \mathrm{~h}$. Thereafter, Ang II was added at different concentrations for various periods of time as indicated. The cells were then collected at different time points for further characterization.

\section{Human renal biopsy specimens}

Renal biopsy specimens were obtained from patients undergoing diagnostic evaluation at the Division of Nephrology,The Second Affiliated Hospital of Nanjing Medical University. A total of 6 subjects were selected from our database.All biopsy specimens were evaluated by a single pathologist who was un aware of the resultsof molecular studies. The patients $(n=3)$ with mild tubulointerstitial fibrosis whose serum creatinine were about $77 \pm 12.4 \mu \mathrm{mol} / \mathrm{l}$ were choosen as a control group; The patients $(\mathrm{n}=3)$ with obvious tubulointerstitial fibrosis whose serum creatinine were about $160 \pm 28.5 \mu \mathrm{mol} / \mathrm{l}$ were choosen as a renal fibrosis group.

\section{MTT assay}

Cells were transfected with the miR-221 mimics or inhibitors and Ets-1 siRNA or negative control for 24 hours before the treatment of Ang II at different concentrations and then seeded at $1 \times 10^{3}$ cells per well in 96-well plates. After a further culture of 24-72h, MTT solution (dissolved in phosphate buffer saline) was added to the wells containing cells (final concentration $0.5 \mathrm{mg} / \mathrm{ml}$ ). The cells were incubated for $4 \mathrm{~h}$ and the medium was removed from the cells and then an aliquot was added to formazan dissolved in $0.1 \%$ dimethylsulfoxide (DMSO) $150 \mu \mathrm{l}$. The absorbance was measured at $490 \mathrm{~nm}$ using a microplate reader and the arithmetic mean OD of 10 wells for each group was calculated. The experiment was repeated at least 3 times.

\section{Boyden chamber for motility assay}

Cells were transfected with the miR-221 mimics or inhibitors and Ets-1 siRNA or negative control for 24 hours and then added onto the upper chamber of the transwell insert ( $8 \mu \mathrm{m}$, Corning-Costar Corp, Corning, New York, USA). After $24 \mathrm{~h}$ of incubation with or without Ang II in the lower chamber at $37^{\circ} \mathrm{C}$, filters were fixed with $3 \%$ paraformaldehyde in PBS and stained with $0.1 \%$ Coomassie blue in $10 \%$ methanol and $10 \%$ actic acid, and the upper surface of the filters was carefully wiped with a cotton-tipped applicator. Cells that migrated across the Transwell filter pores were counted in 5 nonoverlapping $\times 10$ fields and photographed with a Nikon microscope. The experiments were performed in triplicate.

\section{Western Blot}

The preparation of whole-cell lysates and kidney tissue homogenates and western blot analysis of protein expression were carried out using previous routine procedures [24]. The primary antibodies used were as follows: anti- $\alpha$-smooth muscle actin (A5691, Sigma-Aldrich), anti-fibronectin (F3648, Sigma-Aldrich), anti-Ets-1(sc-350, Santa Cruz), and anti-actin (A5385; Sigma Aldrich). Quantification was performed by measurement of the intensity of the signals with aid of National Institutes of Health Image software package. 


\section{Cellular Physiology and Biochemistry}

Cell Physiol Biochem 2014;34:1063-1074

Di et al.: miR-221 Antagonizes Ang II-Induced Renal Fibrosis by Targeting Ets-1

RNA transfection

MiR-221 mimics or inhibitors and their negative control RNA (purchased from Qiagen), as well as Ets-1 small interfering (si) RNA or negative control (Integrated Biotech Solutions, Shanghai, China), were transfected into NRK-49F cells using Liptofectamine 2000 reagent (Invitrogen) following the protocols provided by the manufacturer. After transfection, cells were incubated at $37^{\circ} \mathrm{C}$ in a $\mathrm{CO} 2$ incubator for $24 \mathrm{~h}$ until they were ready for further treatment or assay.

\section{Quantitative polymerase chain reaction}

Total RNA was prepared using a TRIzol RNA isolation system according to the instructions by the manufacturer (Invitrogen).The first strand of cDNA was synthesized using $2 \mu \mathrm{g}$ of RNA in $20 \mu \mathrm{l}$ of reaction buffer using MLV-RT (Promega, Madison, WI).Quantitative polymerase chain reaction (Q-PCR) was performed using an Applied Biosystems 7300 Sequence Detection system. The expression levels of target miRNAs were normalized to U6.The CT data were determined using default threshold settings and the mean CT was determined from the duplicate PCRs. The ratio to sham-control group was calculated by using the equation $2^{-\triangle C T}$, in which $\triangle \mathrm{CT}=\mathrm{CT}$ Sample-CT control. The sequences of primer pairs were as follows: Ets1(forward) 5`-GGGTGGGAGGAAAACAGTTT -3`and (reverse) 5`- CCTTCCATTAAAGTC- CATCTTTGTT -3`; b-Actin (forward) 5`-CAG CTG AGA GGG AAA TCG TG-3` and (reverse) 5`-CGT TGC CAA TAG TGA TGA CC-3`. The PCR products were size fractionated on a 1\% agarose gel and detected by NA-green (D0133, Beyotime) staining. All the primers were acquired from QIAGEN.

\section{Immunohistochemical and Immunofluorescence Staining}

Immunohistochemical staining of kidney sections was performed according to an established protocol [24]. In brief, paraffin-embedded sections were stained with anti-Ets-1(sc-350, Santa Cruz) using the Vector M.O.M. immunodetection kit, according to the protocol by the manufacturer (Vector Laboratories, Burlingame, CA). Indirect immunofluorescence staining was carried out according to the procedures described previously [24]. Briefly, cells were incubated with the specific primary antibodies described above, followed by staining with FITC or TRITC-conjugated secondary antibody.Cells were double stained with DAPI to visualize the nuclei. Slides were viewed with a Nikon Eclipse E80i microscope equipped with a digital camera (DS-Ri1, Nikon). In each experimental setting, immunofluorescence images were captured with identical light exposure parameters.

\section{Morphological Studies}

Tissue sections from the mice were prepared at $3 \mu \mathrm{m}$ thickness by routine procedure. Sections were stained with hematoxylin/eosin or periodic acid Schiff for general histology. Slides were viewed with a Nikon Eclipse E80i microscope equipped with a digital camera (DS-Ri1, Nikon).

\section{Statistical analysis}

Western blotting, RT-PCR, and immunofluorescence staining were all repeated at least three times independently. For Western blot analysis, quantitation was performed by scanning and analyzing the intensity of the hybridization signals using NIH I ImageJ software. Data collected were expressed as mean \pm SEM. Statistical analyses of data were performed using Sigma Stat software (Jandel Scientific Software). Comparison between groups was made using one-way ANOVA, followed by the $t$ test. $\mathrm{P}<0.05$ was considered significant.

\section{Results}

Ets-1 Expression is induced in the fibrotic kidney induced by Ang II

To confirm the role of Ets-1 in Ang II induced renal interstitial fibrosis, we tested the Ets-1 expression in Ang II plasmid injected mice. Compared with pcDNA3 injection, Ets-1 protein level was increased in kidney tissues of the Ang II plasmid injection group (Fig. 1A and B). Immunohistochemical staining revealed that Ang II plasmid injection induced Ets1protein expression both in glomerular and renal interstitium (Fig. 1C though E). Consistent with the western blot and immunohistochemical staining results, Ets-1mRNA was increased after Ang II plasmid injection compared with those injected with empty vector (Fig. 1F). We 
Fig. 1. Ang II increases Ets-1 Expression in vivo. (A) Female Balb/c mice were injected with Ang II expression plasmid through the tail vein. Western blot analysis shows the results of Ets-1 proteins expression in the kidneys of each group. Representative pictures show the results of four animals per group. (B) Graphic presentation of relative Ets-1 abundance normalized to actin. ${ }^{*} \mathrm{P}<0.05$ versus control mice injected with pcDNA3. (C) Representative micrographs show kidney morphology by $\mathrm{H} / \mathrm{E}$ staining. (D) Representative photomicrographs of sections stained with periodic acid Schiff. (E) Immunohistochemical staining of the localization of Ets-1 in kidneys. (F) Quantitative real-time PCR analysis for Ets-1mRNA. * $\mathrm{P}<0.05$ versus control mice injected with pcDNA3. ( $\mathrm{G}$ and $\mathrm{H}$ ) Immunohistochemical staining of the localization of Ets-1 in Renal biopsy specimens.
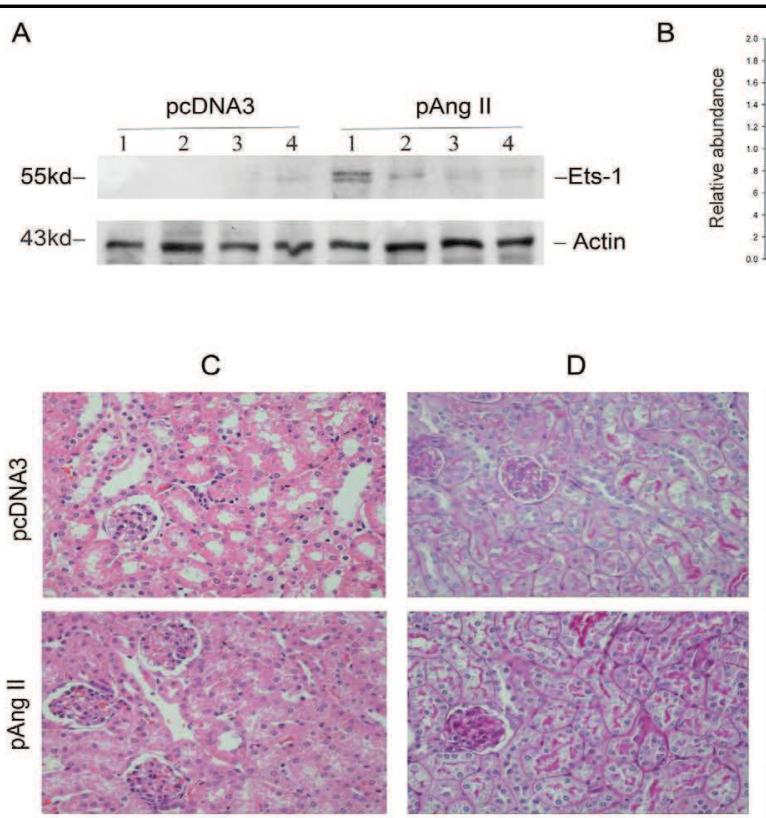

$\mathrm{F}$

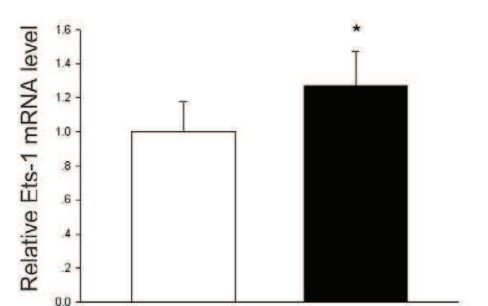

pcDNA3

pAng II

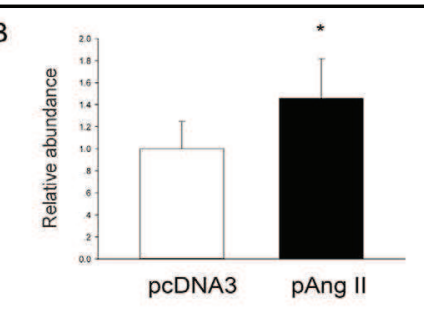

E
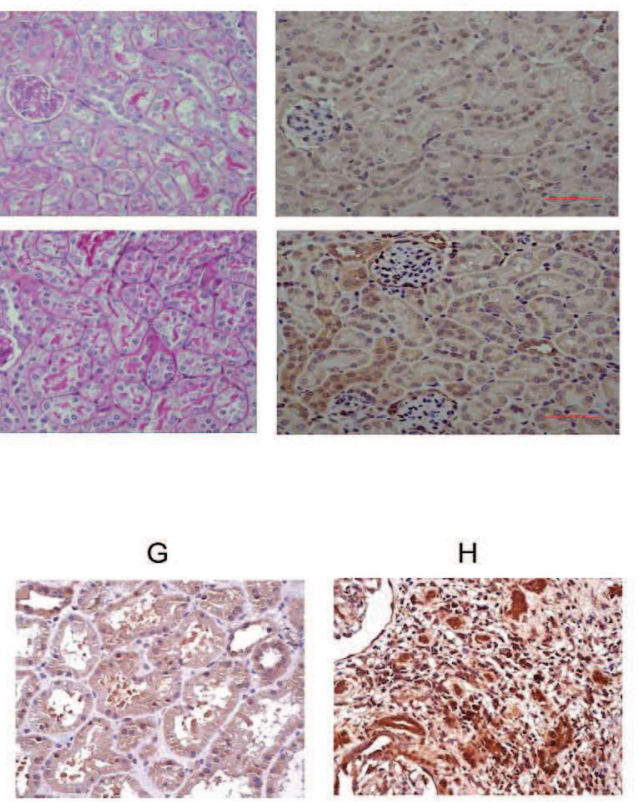

G

$\mathrm{H}$

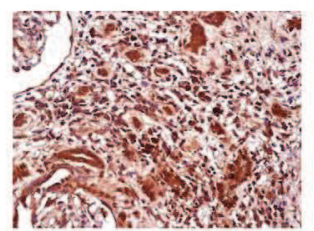

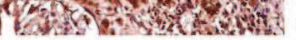

also detected Ets-1 expression in Renal biopsy specimens. Immunohistochemical staining of Ets-1 in the renal biopsies demonstrated the upregulation of Ets- 1 in the obvious renal fibrosis group (Fig. 1H) compared with mild tubulointerstitial fibrosis group (Fig. 1G).

\section{Ang II induces the activation of NRK-49F cells}

Activation of fibroblasts is the central event in AngII induced renal fibrosis. These activated fibroblasts had biological functional changes, and demonstrated by $\alpha$-SMA expression, extracellular matrix (ECM) production,enhanced cell proliferation and migration. To determine the effects of Ang II on proliferation and migration of NRK-49F cells, cells were exposed to Ang II at different concentrations as indicated, and cell survival was assessed by the MTT assay. As shown in Fig. 2A, Ang II induced cell proliferation in a dose-dependent manner. Few pores in Transwell filters were filled with cell extensions after 2 days (Fig. 2B) whereas cells displayed a much more migratory property after incubating of Ang II (Fig. 2C). Next we investigated the effects of Ang II on FN and $\alpha$-SMA expressions. As shown in Fig. 2 ( $\mathrm{E}$ and F), Ang II markedly induced FN and $\alpha$-SMA expressions in NRK-49F cells both in time and dose dependent manner. Similar results were also obtained in immunofluorescent staining assay Fig. 2 ( $\mathrm{G}$ and $\mathrm{H}$ ). 
Fig. 2. Ang II induces the activation of NRK49F cells. (A) MTT assay showing proliferation of NRK-49F cells after different concertration of Ang II treatment. (B and C) Boyden chamber for motility assay showing enhanced migration of NRK-49F cells after Ang II treatment. B: incubation without Ang II. C: incubation with Ang II. (D)Graphic presentation of the numbers of cells or cell extensions migrated through the pores of the filters after incubation with or without Ang II for 2 days. The data are presented as mean \pm S.E. of three experiments. $* \mathrm{P}<0.05$ versus control. (E)Western blot showing FN and $\alpha$-SMA expression in the NRK-49F cells after different time of Ang II treatment. (F) Western blot showing FN and $\alpha$-SMA expression in the NRK-49F cells after diffe-

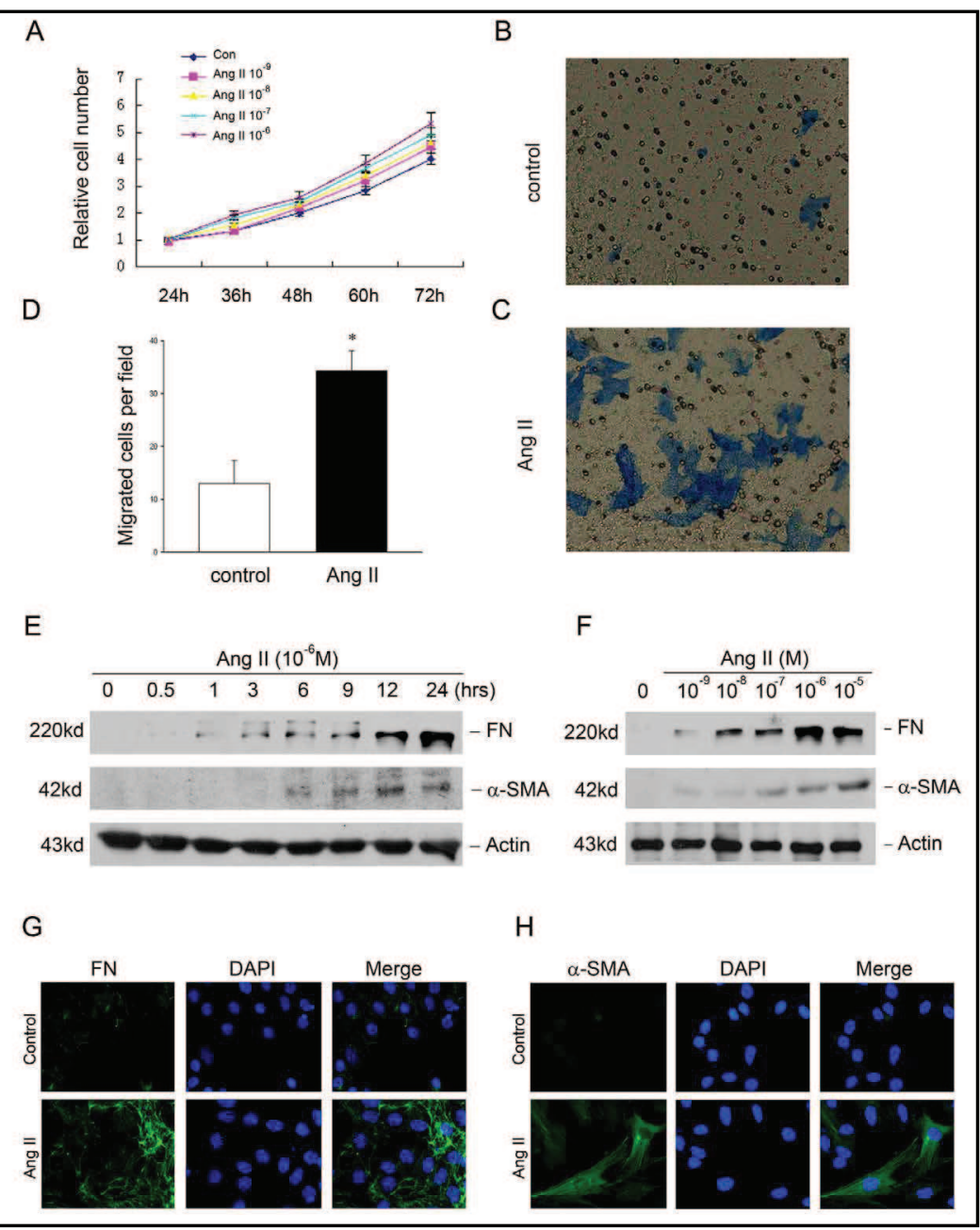

rent concertration of Ang II treatment. (G) Representative micrographs of immunofluorescent staining of FN in NRK-49F cells. (H) Representative micrographs of immunofluorescent staining of $\alpha$-SMA in NRK-49F cells.

Ang II induces Ets-1 expression in NRK-49F cells

In vivo experiment, correlation was found between Ets-1and renal fibrosis. It would be interesting to investigate whether Ets-1 is induced in cultured NRK-49F cells after Ang II treatment. Our results suggested that Ets-1 mRNA expression was obviously increased after Ang II treatment in both time-dependent and dose-dependent manner except at the dose of $10^{-5} \mathrm{M}$ Fig. 3 (A and B). Consistent with the results of quantitative real-time PCR, western blot analysis showed that Ets-1 protein was also increased at $3 \mathrm{~h}$ after Ang II treatment and maintained a high level $24 \mathrm{~h}$ after treatment (Fig. 3C). Ang II induced Ets-1 protein expression in a dose-dependent manner in a range of $0-10^{-6} \mathrm{M}$ (Fig. 3D). Similar results were also obtained in immunofluorescent staining assay. Resting NRK-49F cells expressed little Ets-1 whereas Ang II stimulated NRK-49F cells displayed abundant Ets-1 in the cytoplasm and nucleus (Fig. 3G). Therefore, Ets-1 expression was induced after Ang II treatment in NRK-49F cells.

Knockdown of Ets-1 attenuates Ang II-induced activation of NRK-49F cells

To verify the role of Ets-1 in Ang II induced fibroblast activation. We examined whether knockdown of Ets-1 acted to repress Ang II-induced cell proliferation,migration and FN, $\alpha$-SMA expression. As shown in Fig. 4A and 4B, immunoblotting and immunofluorescent 
Fig. 3. Ang II induces Ets-1 expression in NRK-49F cells. (A and B) Quantitative realtime PCR analysis was used for Ets-1mRNA analysis.A: after different time of Ang II treatment.B: after different concertration of Ang II treatment. (C and D)Western blot analysis was used for showing Ets-1 expression. C: after different time of Ang II treatment. D: after different concertration of Ang II treatment. (E and F) Graphic presentation of relative Ets-1 abundance normalized to actin. D: after different time of Ang II treatment. F: after different concertration of Ang II treatment. (G) Representative immunofluorescent staining images for Ets-1 in NRK-49F cells. The data are presented as mean \pm S.E. of three experiments. * $\mathrm{P}<0.05$ versus control.

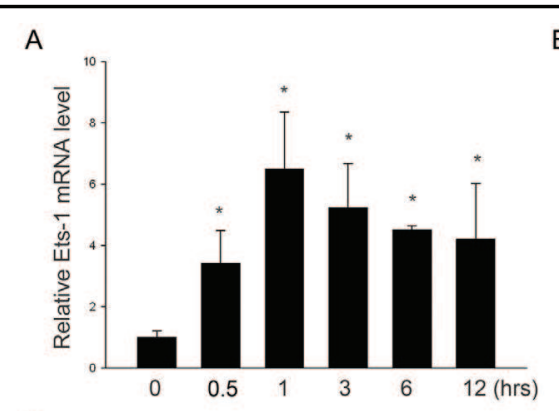

C

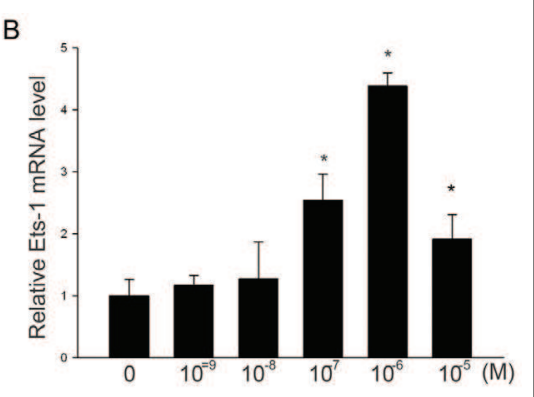

D

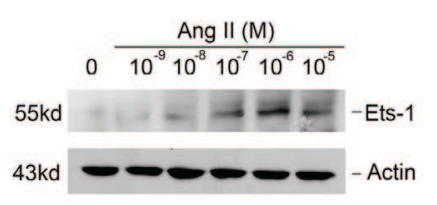

E
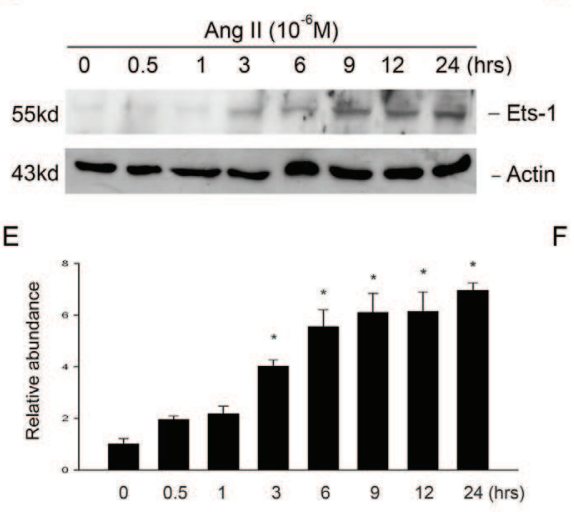

F

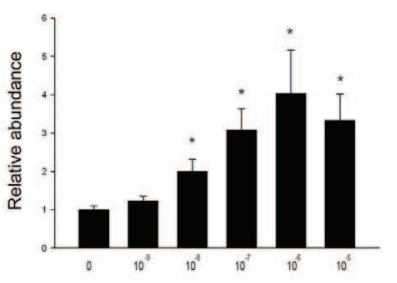

G

Ets-1
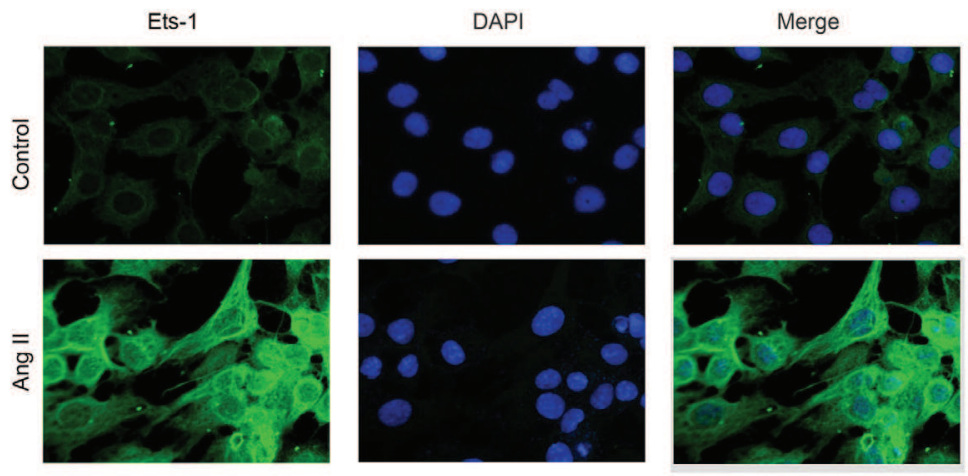

staining showed that the protein level of Ets-1 was significantly down-regulated in cells transfected with Ets-1 siRNA. Fig. 4C shows the result of Ets-1 mRNA levels in NRK-49F cells by Q-PCR analysis, consistent with the western blot results. In addition, Ets-1 knockdown significantly reduced cell proliferation induced by Ang II (Fig. 4 D). The migration of NRK-49F cells induced by Ang II was also inhibited by knockdown of Ets-1 (Fig. 4E- F). Furthermore, inhibition of Ets-1 abolished FN and $\alpha$-SMA expressions induced by Ang II (Fig. 4G-H), suggesting Ets-1 is required for NRK-49F cells activation. Similar results were also obtained in immunofluorescent staining assay (Fig. 6 I). In a word, these results suggested that Ets-1 was involved in Ang II-induced activation of NRK-49F cells.

\section{Ang II induces down-regulation of miR-221 in vivo and in vitro}

Our results indicated that Ets-1 has a critical role in mediating Ang II induced renal fibrosis, but how it is regulated remains obscure. Since TargetScan predicted the existence of evolutionarily conserved binding sites for miR-221 in the 3' untranslated region (UTR) of Ets-1 (Fig. 5A) and it was confirmed by luciferasereport in obesity [25]. So we examined the miR-221 expression in fibrotic kidney induced by Ang II. Quantitative real-time PCR results indicated that miR-221 expression was down regulated both in mice treated with AngII infusion via osmotic minipump and mice injected with Ang II plasmid (Fig. 5B-C). Next we 
Fig. 4. Knockdown of Ets1 attenuates Ang II-induced activation of NRK-49F cells.Western blot analysis (A) and immunofluorescent staining (B) showing Ets-1 protein abundance after Ets-1small interfering RNA (siRNA) transfection. (C) Q-PCR showing Ets-1 mRNA abundance after Ets-1 siRNA transfection. (D) MTT assay showing proliferation of NRK-49F cells. (E) Graphic presentation of the numbers of cells or cell extensions migrated through the pores of the filters. * $\mathrm{P}<0.05$ versus control. (F) Boyden chamber for motility assay showing reduced migration of NRK49F cells after Ets-1small interfering RNA (siRNA) transfection. (G) Western blot analysis showing FN and $\alpha$-SMA expression. (H) Graphic presentation of relative FN and $\alpha$-SMA abundance normalized to actin. ${ }^{*} \mathrm{P}<0.05$ versus control. (I) Representative immunofluorescent staining images for FN and $\alpha$-SMA in NRK-49F cells.
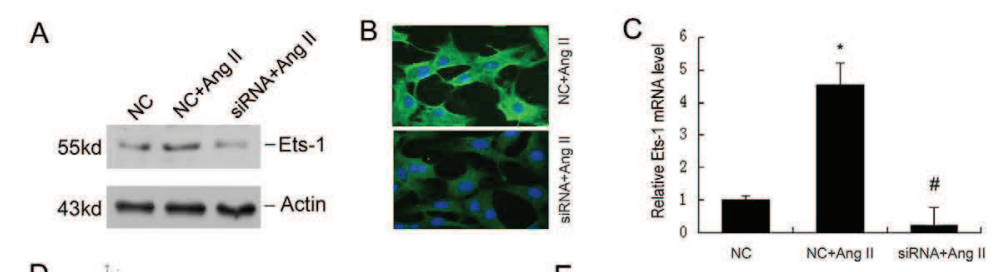

D

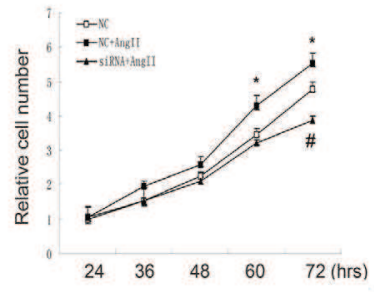

E
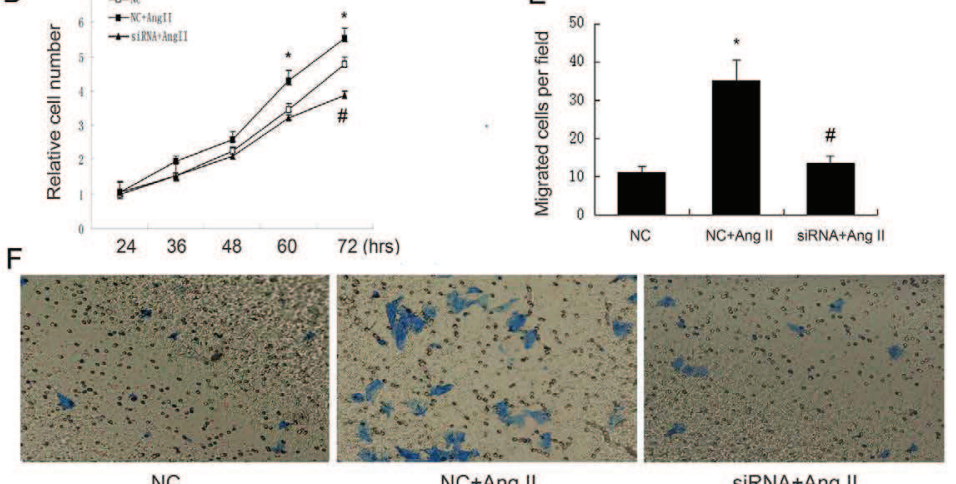

G

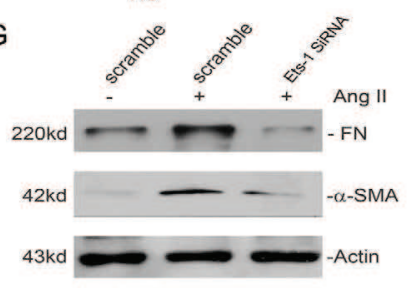

NC+Ang II

siRNA+Ang II

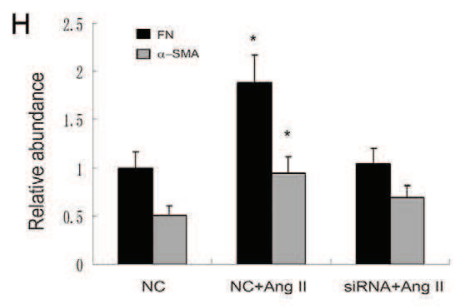

I

NC
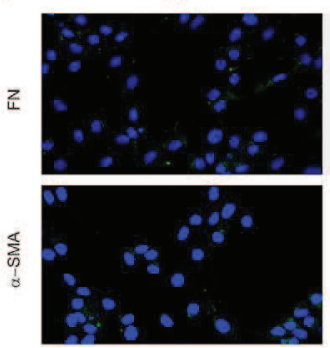

NC+Ang II
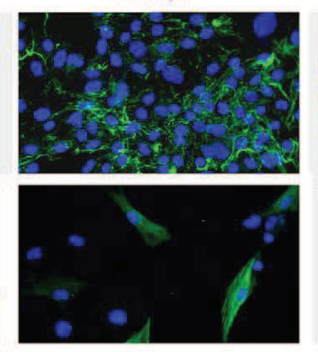

siRnA+Ang II

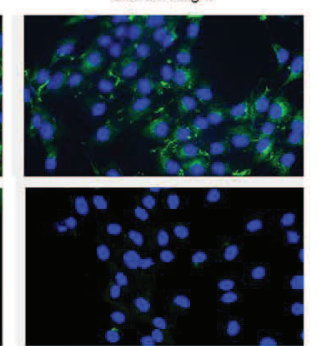

investigated miR-221 expression in NRK-49F cells after Ang II treatment. As shown in Fig. 5(D and E), miR-221 was significantly decreased in time and dose-dependent manner. We further investigated the role of miR-221 on Ets-1 expression in NRK-49F cells. As shown in Fig. 5 ( $F$ and G), transfection of miR-221 mimic significantly decreased Ang II-induced Ets-1 expression, whereas transfection of miR-221 inhibitor induced Ets-1 expression.

\section{MiR-221/Ets-1 axis is involved into Ang II induced NRK-49F cells activation}

To further investigate the role of miR-221/Ets-1 axis in kidney fibrosis, we observed the role of miR-221 in Ang II induced NRK-49F cells activation. NRK-49F cells were transfected with miR-221 mimic or inhibitor before Ang II treatment. As shown in Fig. 6 (A and B), miR221 mimic transfection resisted Ang II induced migration in NRK-49F cells, whereas miR221 inhibitor promoted this effect. Fig. 6 (C and E) shows that miR-221mimic transfection resisted to Ang II-induced activation of NRK-49F cells, whereas miR-221 inhibitor promoted phenotype changes viewed as induction of $\alpha$-SMA and FN expression Fig. 6 (D and F). From the discussion above, it was indicated miR-221/Ets-1axis is involved in Ang II induced NRK$49 \mathrm{~F}$ cells activation. 
Fig. 5. Ang II induces downregulation of miR-221 in vivo and in vitro (A) Predicted consequential pairing of Ets-1 and miR-221. (B) Male CD1 mice were subcutaneously embedded with a mini pump infused with purified Ang II protein. Quantitative real-time PCR showing miR-221 expression in kidneys. * $\mathrm{P}<0.05$ versus control. (C) Female Balb/c mice were injected with Ang II expression plasmid through the tail vein. Quantitative real-time PCR showing miR221 expression in kidneys. * $\mathrm{P}<0.05$ versus control mice injected with pcDNA3. (D) Quantitative real-time PCR showing miR-221 expression after different time of Ang II treatment in NRK-49F cells. * $\mathrm{P}<0.05$ versus control. (E) Quantitative real-time PCR showing miR-221 and Ets1after different dose of Ang II treatment in NRK-49F cells. * $\mathrm{P}<0.05$ versus control. (F) Western blot analysis showing Ets-1 expression after miR221 miminc or inhibitor treatment in NRK-49F cells with or without Ang II treatment. (G) Graphic presentation of relative Ets-1 abundance normalized to actin after miR-221 miminc or inhibitor treatment in NRK-49F cells with or without Ang II treatment. * $\mathrm{P}<0.05$ versus control. \# $\mathrm{P}<0.05$ versus Ang II treatment.

\section{Discussion}

Renal fibrosis is an inevitable outcome of almost all progressive chronic kidney disease. Maladaptive activation of the Renin-angiotensin-aldosterone system (RAAS) is a key regulator of renal fibrosis [26], and inhibition of RAAS system could attenuate glomerulosclerosis and interstitial fibrosis. As the main effector of RAAS, Ang II has been considered as a renal growth factor with many effects on the kidney as modulating cell growth and extracellular matrix (ECM) synthesis. Accumulated evidences have shown that Ang II is a profibrotic cytokine and acttivation of fibroblast to myofibroblast plays a crucial role in promoting renal fibrosis induced by Ang II $[27,28]$. However, the cellular and molecular mechanisms of this process are still under investigation. In this study, we presented evidence suggesting that Ets-1, targeted by miR-221, acts as an important regulator implicated in the activation of renal fibroblast induced by Ang II.

Many transcription factors are involved in the pathogenesis of CKD and mediate the complex pathways in response to Ang II. Ets- 1 is a member of the Ets family of transcription factors that share a highly conserved DNA-binding domain. Although it is reported to be 


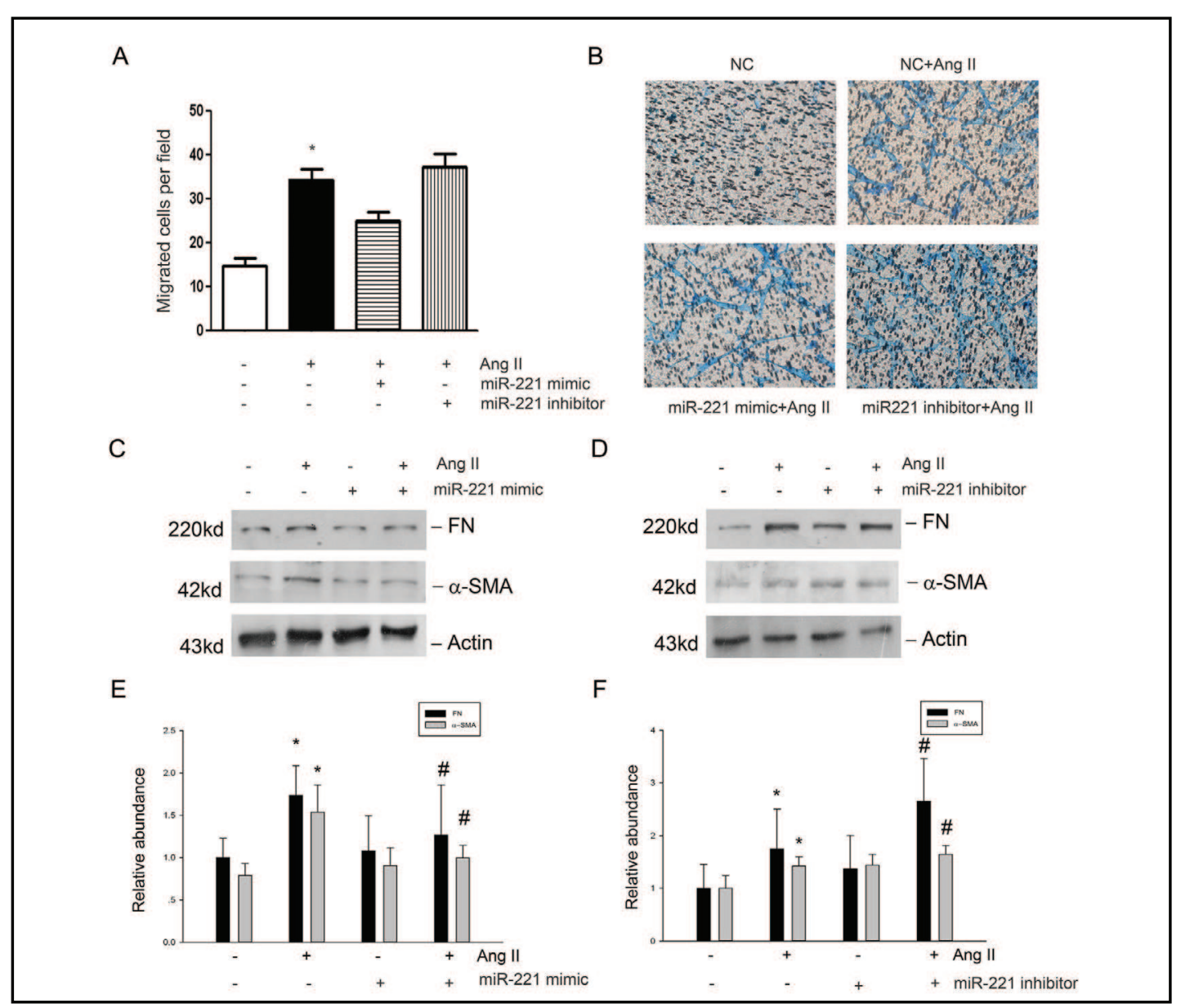

Fig. 6. MiR-221/Ets-1 axis is involved into Ang II induced NRK-49F cells. (A) Graphic presentation of the numbers of cells or cell extensions migrated through the pores of the filters after miR-221-mimic or miR221-inhibitor transfection along with Ang II treatment (B) Boyden chamber for motility assay showing migration of NRK-49F cells after miR-221-mimic or miR-221-inhibitor transfection along with Ang II treatment. (C) Western blot analysis showing miR-221-miminc transfection reduced FN and $\alpha$-SMA expression. (D) Western blot analysis showing miR-221-inhibitor transfection increased FN and $\alpha$-SMA expression. (E) Graphic presentation of relative FN and $\alpha$-SMA abundance normalized to actin incubated with or without Ang II after miR-221-miminc transfection. (F) Graphic presentation of relative FN and $\alpha$-SMA abundance normalized to actin incubated with or without Ang II after miR-221-inhibitor transfection. $* \mathrm{P}<0.05$ versus control. \# $\mathrm{P}<0.05$ versus Ang II treatment.

required for the normal development of the mammalian kidney, many recent studies have described that Ets-1 is up-regulated in a wide range of renal diseases and regulate numerous genes such as MMP-1, -3, -9, TIMP-1, urokinase-type plasminogen activator(u-PA), MCP-1 and PAI-1 [29-33]. However, most studies mainly focus on glomerular and mesangial cells. The role of Ets-1 in renal interstitial fibroblast activation and tubulointerstitial fibrosis remains largely unknown. Our resulsts displayed that Ets-1 was induced by Ang II in rat renal interstitial fibroblast cells accompanied by fibroblast activation. Knockdown of Ets-1 significantly inhibited this effect. To our knowledge, this is the first report describing the critical role of Ets-1 in Ang II induced interstitial fibroblast activation. More over, we also first described that Ang II plasmid injection could increase Ets-1 expression, which is consistent with the results of Ang II infusion mice model reported by previous study [34]. To further confirm the role of Ets-1 in tubulointerstitial fibrosis, we detected the Ets- 1 expression in renal biopsies of patients with mild or obvious tubulointerstitial fibrosis. Our data suggested 


\section{Cellular Physiology and Biochemistry}

Cell Physiol Biochem 2014;34:1063-1074

\begin{tabular}{l|l}
\hline DOI: 10.1159/000366321 & (c) 2014 S. Karger AG, Basel
\end{tabular}

\begin{tabular}{l|l} 
Published onlıne: September 08, 2014 & www.karger.com/cpb
\end{tabular}

Di et al.: miR-221 Antagonizes Ang II-Induced Renal Fibrosis by Targeting Ets-1

Ets-1 was upregulated in fibrotic renal biopsy specimens. Taken together, our findings would suggest that Ets-1 is an important mediator of Ang II in tubulointerstitial fibrosis.

In previous studies, we have demonstrated that miR-30e and miR-200 family were involved in renal fibrosis [35, 36]. MiR-221 was found under-expressed in Unilateral Ureteral Obstruction(UUO) mouse model using miRNA array [23]. Our further investigation found that miR-221 was downregulated in vivo. Similar results were also obtained in NRK-49F cells treated with Ang II. Therefore it is suggested that miR-221 downregulation might be an important facilitator in renal fibrosis. Target screening and previous luciferase assays have confirmed Ets-1 is the target of miR-221 [25]. In this study, the expression of miR-221 was inversely related to the Ets- 1 expression treated with Ang II in vivo and in vitro. Inhibition of miR-221 increased Ets-1 levels and was accompanied by the activation of NRK-49F similar to Ang II treatment. In contrast, overexpression of miR-221 hindered this phenotypic change by downregulation of Ets-1 expression. Taken together, our results indicate that miR-221 has an important role in mediating Ang II-induced interstitial myofibroblast activation and kidney fibrosis by target Ets-1. These findings may provide new therapeutic targets in fibrotic kidney diseases.

\section{References}

1 McFarlane SI, McCullough PA, Sowers JR, Soe K, Chen SC, Li S, Vassalotti JA, Stevens LA, Salifu MO, Kurella Tamura M, Bomback AS, Norris KC, Collins AJ, Bakris GL, Whaley-Connell AT: Comparison of the ckd epidemiology collaboration (ckd-epi) and modification of diet in renal disease (mdrd) study equations: Prevalence of and risk factors for diabetes mellitus in ckd in the kidney early evaluation program (keep). Am J Kidney Dis 2011;57:S24-31.

- Kovesdy CP, Kalantar-Zadeh K: Enter the dragon: A chinese epidemic of chronic kidney disease? Lancet 2012;379:783-785.

-3 Wynn TA, Ramalingam TR: Mechanisms of fibrosis: Therapeutic translation for fibrotic disease. Nat Med 2012;18:1028-1040.

4 Strutz F, Zeisberg M: Renal fibroblasts and myofibroblasts in chronic kidney disease. J Am Soc Nephrol 2006;17:2992-2998.

5 Liu Y: Cellular and molecular mechanisms of renal fibrosis. Nat Rev Nephrol 2011;7:684-696.

6 Hu K, Lin L, Tan X, Yang J, Bu G, Mars WM, Liu Y: Tpa protects renal interstitial fibroblasts and myofibroblasts from apoptosis. J Am Soc Nephrol 2008;19:503-514.

7 Boor P, Ostendorf T, Floege J: Renal fibrosis: Novel insights into mechanisms and therapeutic targets. Nat Rev Nephrol 2010;6:643-656.

-8 Hinz B: The myofibroblast: Paradigm for a mechanically active cell. J Biomech 2010;43:146-155.

9 He W, Dai C, Li Y, Zeng G, Monga SP, Liu Y: Wnt/beta-catenin signaling promotes renal interstitial fibrosis. J Am Soc Nephrol 2009;20:765-776.

10 Xia Y, Entman ML, Wang Y: Critical role of cxcl16 in hypertensive kidney injury and fibrosis. Hypertension 2013;62:1129-1137.

-11 Mezzano SA, Ruiz-Ortega M, Egido J: Angiotensin ii and renal fibrosis. Hypertension 2001;38:635-638.

12 Russell L, Garrett-Sinha LA: Transcription factor ets-1 in cytokine and chemokine gene regulation. Cytokine 2010;51:217-226.

13 Razzaque MS, Naito T, Taguchi T: Proto-oncogene ets-1 and the kidney. Nephron 2001;89:1-4.

14 Oikawa T, Yamada T: Molecular biology of the ets family of transcription factors. Gene 2003;303:11-34.

-15 Ni W, Zhan Y, He H, Maynard E, Balschi JA, Oettgen P: Ets-1 is a critical transcriptional regulator of reactive oxygen species and p47(phox) gene expression in response to angiotensin ii. Circ Res 2007;101:985-994.

-16 Ebrahimian T, Li MW, Lemarie CA, Simeone SM, Pagano PJ, Gaestel M, Paradis P, Wassmann S, Schiffrin EL: Mitogen-activated protein kinase-activated protein kinase 2 in angiotensin ii-induced inflammation and hypertension: Regulation of oxidative stress. Hypertension 2011;57:245-254.

17 Bartel DP: Micrornas: Genomics, biogenesis, mechanism, and function. Cell 2004;116:281-297. 


\section{Cellular Physiology and Biochemistry}

Cell Physiol Biochem 2014;34:1063-1074

\begin{tabular}{l|l}
\hline DOI: $10.1159 / 000366321$ & (c) 2014 S. Karger AG, Basel
\end{tabular}

\begin{tabular}{l|l} 
Published onIIne: September 08, 2014 & www.karger.com/cpb
\end{tabular}

Di et al.: miR-221 Antagonizes Ang II-Induced Renal Fibrosis by Targeting Ets-1

18 Zhu N, Zhang D, Chen S, Liu X, Lin L, Huang X, Guo Z, Liu J, Wang Y, Yuan W, Qin Y: Endothelial enriched micrornas regulate angiotensin ii-induced endothelial inflammation and migration. Atherosclerosis 2011;215:286-293.

19 Ye X, Bai W, Zhu H, Zhang X, Chen Y, Wang L, Yang A, Jia L: Mir-221 promotes trastuzumab-resistance and metastasis in her2-positive breast cancers by targeting pten. BMB Rep 201;47:268-273.

20 Sun T, Wang X, He HH, Sweeney CJ, Liu SX, Brown M, Balk S, Lee GS, Kantoff PW: Mir-221 promotes the development of androgen independence in prostate cancer cells via downregulation of hectd2 and rab1a. Oncogene 2014;33:2790-2800.

21 Rong M, Chen G, Dang Y: Increased mir-221 expression in hepatocellular carcinoma tissues and its role in enhancing cell growth and inhibiting apoptosis in vitro. BMC Cancer 2013;13:21.

-22 Lupini L, Bassi C, Ferracin M, Bartonicek N, D'Abundo L, Zagatti B, Callegari E, Musa G, Moshiri F, Gramantieri L, Corrales FJ, Enright AJ, Sabbioni S, Negrini M: Mir-221 affects multiple cancer pathways by modulating the level of hundreds messenger rnas. Front Genet 2013;4:64.

23 Glowacki F, Savary G, Gnemmi V, Buob D, Van der Hauwaert C, Lo-Guidice JM, Bouye S, Hazzan M, Pottier N, Perrais M, Aubert S, Cauffiez C: Increased circulating mir-21 levels are associated with kidney fibrosis. PLoS One 2013;8:e58014.

-24 Yang Z, Xiaohua W, Lei J, Ruoyun T, Mingxia X, Weichun H, Li F, Ping W, Junwei Y: Uric acid increases fibronectin synthesis through upregulation of lysyl oxidase expression in rat renal tubular epithelial cells. Am J Physiol Renal Physiol 2010;299:F336-346.

25 Meerson A, Traurig M, Ossowski V, Fleming JM, Mullins M, Baier LJ: Human adipose microrna-221 is upregulated in obesity and affects fat metabolism downstream of leptin and tnf-alpha. Diabetologia 2013;56:1971-1979.

-26 Mezzano SA, Aros CA, Droguett A, Burgos ME, Ardiles LG, Flores CA, Carpio D, Vio CP, Ruiz-Ortega M, Egido $\mathrm{J}$ : Renal angiotensin ii up-regulation and myofibroblast activation in human membranous nephropathy. Kidney Int 2003:S39-45.

27 Macconi D: Targeting the renin angiotensin system for remission/regression of chronic kidney disease. Histol Histopathol 2010;25:655-668.

28 Ruster C, Wolf G: Angiotensin ii as a morphogenic cytokine stimulating renal fibrogenesis. J Am Soc Nephrol 2011;22:1189-1199.

-29 Naito T, Razzaque MS, Nazneen A, Liu D, Nihei H, Koji T, Taguchi T: Renal expression of the ets-1 protooncogene during progression of rat crescentic glomerulonephritis. J Am Soc Nephrol 2000;11:2243-2255.

30 Kanazawa A, Tsukada S, Sekine A, Tsunoda T, Takahashi A, Kashiwagi A, Tanaka Y, Babazono T, Matsuda M, Kaku K, Iwamoto Y, Kawamori R, Kikkawa R, Nakamura Y, Maeda S: Association of the gene encoding wingless-type mammary tumor virus integration-site family member $5 \mathrm{~b}$ (wnt5b) with type 2 diabetes. Am J Hum Genet 2004;75:832-843.

31 Puri S, Rodova M, Islam MR, Magenheimer BS, Maser RL, Calvet JP: Ets factors regulate the polycystic kidney disease-1 promoter. Biochem Biophys Res Commun 2006;342:1005-1013.

32 Raffetseder U, Wernert N, Ostendorf T, van Roeyen C, Rauen T, Behrens P, Floege J, Mertens PR: Mesangial cell expression of proto-oncogene ets-1 during progression of mesangioproliferative glomerulonephritis. Kidney Int 2004;66:622-632.

33 Liu DX, Liu XM, Su Y, Zhang XJ: Renal expression of proto-oncogene ets-1 on matrix remodeling in experimental diabetic nephropathy. Acta Histochem 2011;113:527-533.

-34 Feng W, Chumley P, Hua P, Rezonzew G, Jaimes D, Duckworth MW, Xing D, Jaimes EA: Role of the transcription factor erythroblastosis virus e26 oncogen homolog-1 (ets-1) as mediator of the renal proinflammatory and profibrotic effects of angiotensin ii. Hypertension 2012;60:1226-1233.

-35 Jiang L, Qiu W, Zhou Y, Wen P, Fang L, Cao H, Zen K, He W, Zhang C, Dai C, Yang J: A microrna-30e/ mitochondrial uncoupling protein 2 axis mediates tgf-beta1-induced tubular epithelial cell extracellular matrix production and kidney fibrosis. Kidney Int 2013;84:285-296.

-36 Xiong M, Jiang L, Zhou Y, Qiu W, Fang L, Tan R, Wen P, Yang J: The mir-200 family regulates tgf-beta1induced renal tubular epithelial to mesenchymal transition through smad pathway by targeting zeb1 and zeb2 expression. Am J Physiol Renal Physiol 2012;302:F369-379. 